\title{
When Conductance is less than the Sum of its Parts: Exploring Interference in Multi-Connected Molecules
}

\author{
Tim Hansen and Gemma C. Solomon* \\ Nano-Science Center and Department of Chemistry, University of Copenhagen \\ Universitetsparken 5, 2100 Copenhagen Ø, Denmark \\ E-mail: gsolomon@nano.ku.dk
}

\section{Supporting Information}

\section{Model Matrix Representation}

To write up a matrix representation of the Green's function necessary for a transmission calculation for a model system, we first divide our full Hamiltonian for the entire system, $\hat{H}$, into a Hamiltonian for the left and a right lead, $\hat{H}_{L}$ and $\hat{H}_{R}$, respectively, and a Hamiltonian for the molecule or device, $\hat{H}_{D}$,

$$
\hat{H}=\left(\begin{array}{ccc}
\hat{H}_{L} & \hat{\tau}_{L}^{\dagger} & 0 \\
\hat{\tau}_{L} & \hat{H}_{D} & \hat{\tau}_{R} \\
0 & \hat{\tau}_{R}^{\dagger} & \hat{H}_{R}
\end{array}\right)
$$

Here $\hat{\tau}_{L / R}$ are the coupling matrices between the electrodes and the molecule and it is these that determine the molecule's connectivity and the coherence between the connections if 
there are more than one per electrode. By invoking the wideband approximation $\hat{H}_{L}$ and $\hat{H}_{R}$ no longer play a role and the construction of the Green's function is straight forward

$$
G(\varepsilon)=\left[\varepsilon \cdot \mathbb{1}-\hat{H}_{D}-\Sigma\right]^{-1},
$$

where $\Sigma$ is the self-energy, here given by

$$
\begin{aligned}
& \Sigma=\frac{-\mathrm{i}}{2}\left(\Gamma_{L}+\Gamma_{R}\right), \\
& \Gamma_{L / R}=\hat{\tau}_{L / R} \cdot \hat{\tau}_{L / R}^{\dagger} .
\end{aligned}
$$

From here the transmission is calculated as the trace

$$
T(\varepsilon)=\operatorname{Tr}\left[\Gamma_{L} G(\varepsilon) \Gamma_{R} G^{\dagger}(\varepsilon)\right] .
$$

For our model calculations the device Hamiltonian looks like

$$
\hat{H}_{D}=\left(\begin{array}{llllll}
\epsilon & g & 0 & 0 & 0 & g \\
g & \epsilon & g & 0 & 0 & 0 \\
0 & g & \epsilon & g & 0 & 0 \\
0 & 0 & g & \epsilon & g & 0 \\
0 & 0 & 0 & g & \epsilon & g \\
g & 0 & 0 & 0 & g & \epsilon
\end{array}\right),
$$

where $\epsilon$ is the site energy of the molecular sites relative to the Fermi energy and $g$ is the hopping element. Going through the cases for the molecules based on the ortho configuration (left binding groups on sites 1 and 3, right binding groups on sites 4 and 6, with numbering increasing in a clock-wise direction) the coupling matrices, first for the direct connection are 
given by

$$
\hat{\tau}_{L}=\left(\begin{array}{l}
0 \\
0 \\
\tau \\
0 \\
0 \\
0
\end{array}\right), \quad \hat{\tau}_{R}=\left(\begin{array}{l}
0 \\
0 \\
0 \\
\tau \\
0 \\
0
\end{array}\right),
$$

where $\tau$ is the coupling strength. For the crossed connection

$$
\hat{\tau}_{L}=\left(\begin{array}{l}
0 \\
0 \\
\tau \\
0 \\
0 \\
0
\end{array}\right), \quad \hat{\tau}_{R}=\left(\begin{array}{l}
0 \\
0 \\
0 \\
0 \\
0 \\
\tau
\end{array}\right) .
$$

Finally for the spider connection we first look at the coherent case

$$
\hat{\tau}_{L}=\left(\begin{array}{c}
\tau \\
0 \\
\tau \\
0 \\
0 \\
0
\end{array}\right), \quad \hat{\tau}_{R}=\left(\begin{array}{l}
0 \\
0 \\
0 \\
\tau \\
0 \\
\tau
\end{array}\right),
$$

and then the incoherent 


$$
\hat{\tau}_{L}=\left(\begin{array}{cc}
\tau & 0 \\
0 & 0 \\
0 & \tau \\
0 & 0 \\
0 & 0 \\
0 & 0
\end{array}\right), \quad \hat{\tau}_{R}=\left(\begin{array}{cc}
0 & 0 \\
0 & 0 \\
0 & 0 \\
\tau & 0 \\
0 & 0 \\
0 & \tau
\end{array}\right)
$$

The resulting gammas are, for the directly connected molecule

$$
\Gamma_{L}=\left(\begin{array}{cccccc}
0 & 0 & 0 & 0 & 0 & 0 \\
0 & 0 & 0 & 0 & 0 & 0 \\
0 & 0 & \tau^{2} & 0 & 0 & 0 \\
0 & 0 & 0 & 0 & 0 & 0 \\
0 & 0 & 0 & 0 & 0 & 0 \\
0 & 0 & 0 & 0 & 0 & 0
\end{array}\right), \quad \Gamma_{R}=\left(\begin{array}{cccccc}
0 & 0 & 0 & 0 & 0 & 0 \\
0 & 0 & 0 & 0 & 0 & 0 \\
0 & 0 & 0 & 0 & 0 & 0 \\
0 & 0 & 0 & \tau^{2} & 0 & 0 \\
0 & 0 & 0 & 0 & 0 & 0 \\
0 & 0 & 0 & 0 & 0 & 0
\end{array}\right),
$$

the crossed

$$
\Gamma_{L}=\left(\begin{array}{cccccc}
0 & 0 & 0 & 0 & 0 & 0 \\
0 & 0 & 0 & 0 & 0 & 0 \\
0 & 0 & \tau^{2} & 0 & 0 & 0 \\
0 & 0 & 0 & 0 & 0 & 0 \\
0 & 0 & 0 & 0 & 0 & 0 \\
0 & 0 & 0 & 0 & 0 & 0
\end{array}\right), \quad \Gamma_{R}=\left(\begin{array}{llllll}
0 & 0 & 0 & 0 & 0 & 0 \\
0 & 0 & 0 & 0 & 0 & 0 \\
0 & 0 & 0 & 0 & 0 & 0 \\
0 & 0 & 0 & 0 & 0 & 0 \\
0 & 0 & 0 & 0 & 0 & 0 \\
0 & 0 & 0 & 0 & 0 & \tau^{2}
\end{array}\right),
$$

the coherent spider 


$$
\Gamma_{L}=\left(\begin{array}{cccccc}
\tau^{2} & 0 & \tau^{2} & 0 & 0 & 0 \\
0 & 0 & 0 & 0 & 0 & 0 \\
\tau^{2} & 0 & \tau^{2} & 0 & 0 & 0 \\
0 & 0 & 0 & 0 & 0 & 0 \\
0 & 0 & 0 & 0 & 0 & 0 \\
0 & 0 & 0 & 0 & 0 & 0
\end{array}\right), \quad \Gamma_{R}=\left(\begin{array}{cccccc}
0 & 0 & 0 & 0 & 0 & 0 \\
0 & 0 & 0 & 0 & 0 & 0 \\
0 & 0 & 0 & 0 & 0 & 0 \\
0 & 0 & 0 & \tau^{2} & 0 & \tau^{2} \\
0 & 0 & 0 & 0 & 0 & 0 \\
0 & 0 & 0 & \tau^{2} & 0 & \tau^{2}
\end{array}\right),
$$

and the incoherent spider

$$
\Gamma_{L}=\left(\begin{array}{cccccc}
\tau^{2} & 0 & 0 & 0 & 0 & 0 \\
0 & 0 & 0 & 0 & 0 & 0 \\
0 & 0 & \tau^{2} & 0 & 0 & 0 \\
0 & 0 & 0 & 0 & 0 & 0 \\
0 & 0 & 0 & 0 & 0 & 0 \\
0 & 0 & 0 & 0 & 0 & 0
\end{array}\right), \quad \Gamma_{R}=\left(\begin{array}{cccccc}
0 & 0 & 0 & 0 & 0 & 0 \\
0 & 0 & 0 & 0 & 0 & 0 \\
0 & 0 & 0 & 0 & 0 & 0 \\
0 & 0 & 0 & \tau^{2} & 0 & 0 \\
0 & 0 & 0 & 0 & 0 & 0 \\
0 & 0 & 0 & 0 & 0 & \tau^{2}
\end{array}\right) .
$$

Setting the coupling elements for the spider connection in the coherent case to be the same may be an oversimplification of the problem. If we allow the argument in the complex plane of these coupling elements to differ we end up with

$$
\hat{\tau}_{L}=\left(\begin{array}{c}
\tau \\
0 \\
\tau \cdot \mathrm{e}^{\mathrm{i} \theta} \\
0 \\
0 \\
0
\end{array}\right), \quad \hat{\tau}_{R}=\left(\begin{array}{c}
0 \\
0 \\
0 \\
\tau \cdot \mathrm{e}^{\mathrm{i} \theta} \\
0 \\
\tau
\end{array}\right)
$$




$$
\Gamma_{L}=\left(\begin{array}{cccccc}
\tau^{2} & 0 & \tau^{2} \cdot \mathrm{e}^{-\mathrm{i} \theta} & 0 & 0 & 0 \\
0 & 0 & 0 & 0 & 0 & 0 \\
\tau^{2} \cdot \mathrm{e}^{\mathrm{i} \theta} & 0 & \tau^{2} & 0 & 0 & 0 \\
0 & 0 & 0 & 0 & 0 & 0 \\
0 & 0 & 0 & 0 & 0 & 0 \\
0 & 0 & 0 & 0 & 0 & 0
\end{array}\right), \quad \Gamma_{R}=\left(\begin{array}{cccccc}
0 & 0 & 0 & 0 & 0 & 0 \\
0 & 0 & 0 & 0 & 0 & 0 \\
0 & 0 & 0 & 0 & 0 & 0 \\
0 & 0 & 0 & \tau^{2} & 0 & \tau^{2} \cdot \mathrm{e}^{\mathrm{i} \theta} \\
0 & 0 & 0 & 0 & 0 & 0 \\
0 & 0 & 0 & \tau^{2} \cdot \mathrm{e}^{-\mathrm{i} \theta} & 0 & \tau^{2}
\end{array}\right)
$$

Using this later approach to calculate transmission functions with the argument difference chosen to be $0,1 / 2 \pi, \pi$ and $3 / 2 \pi$, and a similar expression for the meta based molecule, we can plot the transmissions seen in Figure S1.
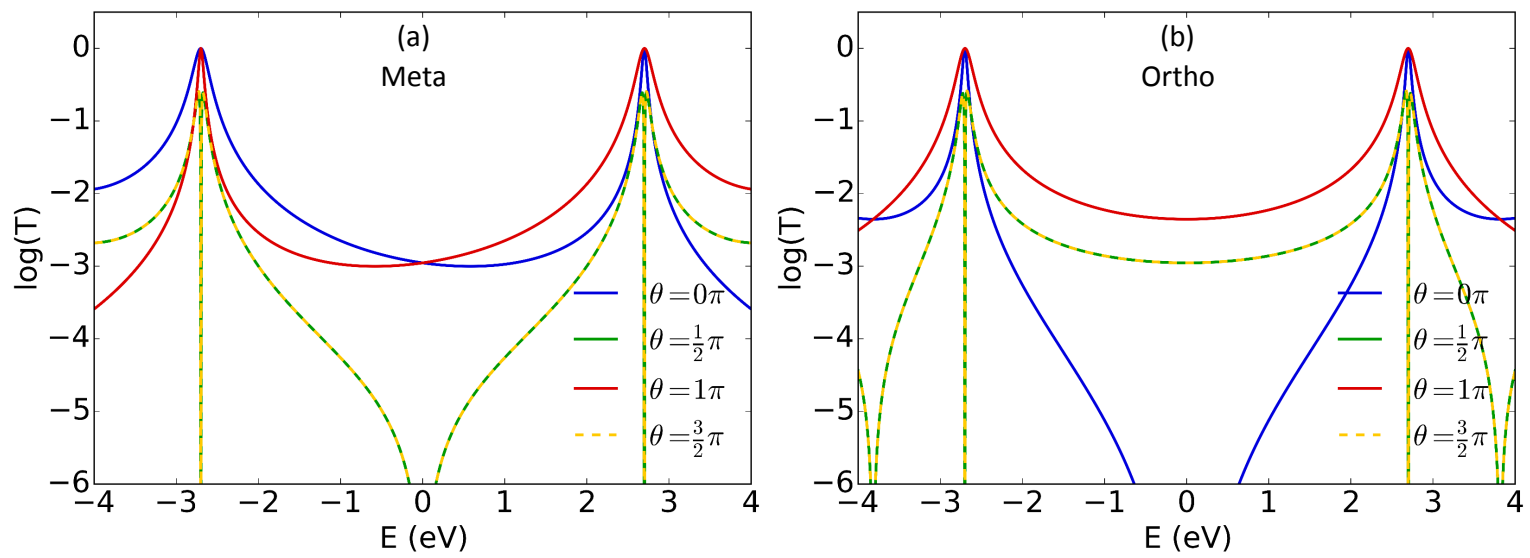

Figure S1: Transmission of coherently connected spider Benzene from model calculations. (a) shows the transmissions based on the meta connected benzene and (b) the ortho connected benzene. The blue line shows the transmission when the argument difference of the coherence is $0 \pi$, the green when it is $1 / 2 \pi$, the red $1 \pi$ and the yellow when it is $3 / 2 \pi$, which have been dashed to allow transparency.

\section{Local \& Channels}

Solomon et al. ${ }^{1}$ have derived an expression for the local current, $I_{m n}$, between atom $m$ and $n$ as 


$$
\begin{aligned}
I_{m n} & =\frac{2 e}{\hbar} \int \frac{\mathrm{d} \varepsilon}{2 \pi} K_{m n}(\varepsilon) \\
K_{m n} & =\sum_{i \in m} \sum_{\substack{j \in n \\
n \neq m}}\left(H_{i j} G_{j i}^{<}(\varepsilon)-H_{j i} G_{i j}^{<}(\varepsilon)\right),
\end{aligned}
$$

where $H$ is the molecular Hamiltonian and $G^{<}$i the lesser Green's function. Here $K_{m n}$ is a Fourier component of the local current, but has the same dimension as transmission. It is this matrix that is plotted in the paper and is referred to as the "local transmission elements". The integrand, $K_{m n}$, can be rewritten as

$$
\begin{aligned}
K_{m n}= & \sum_{i \in m} \sum_{\substack{j \in n \\
n \neq m}} \sum_{k l}\left(\mathrm{i} f_{L}\left(H_{i j} G_{j k}^{r} \Gamma_{k l}^{L} G_{l i}^{a}-H_{j i} G_{i k}^{r} \Gamma_{k l}^{L} G_{l j}^{a}\right)\right)- \\
& \left(\mathrm{i} f_{R}\left(H_{i j} G_{j k}^{r} \Gamma_{k l}^{R} G_{l i}^{a}-H_{j i} G_{i k}^{r} \Gamma_{k l}^{R} G_{l j}^{a}\right)\right) .
\end{aligned}
$$

Gagliardi et al. ${ }^{2}$ have shown that $A^{L / R}=G \Gamma^{L / R} G$ holds information about the channels, which can be obtained by diagonalization. This means it is possible to rewrite $H_{i j} G_{j k}^{r} \Gamma_{k l}^{L / R} G_{l i}^{a}$

$$
\sum_{k l} H_{i j} G_{j k}^{r} \Gamma_{k l}^{L / R} G_{l i}^{a}=H_{i j} A_{j i}^{L / R}=\sum_{\alpha} H_{i j} U_{j \alpha} \bar{A}_{\alpha \alpha}^{L / R} U_{\alpha i}^{\dagger}
$$

where $\bar{A}$ is the diagonalized form of $A$, i.e. $\bar{A}=U^{\dagger} A U$. This now makes it possible to write an expression where the integrand of the local current is split into contributions from the channels of the molecular junction 


$$
\begin{aligned}
K_{m n}= & \sum_{\alpha} K_{m n}^{\alpha} \\
K_{m n}^{\alpha}= & \sum_{i \in m} \sum_{\substack{j \in n \\
n \neq m}}\left(\mathrm{i} f_{L}\left(H_{i j} U_{L, j \alpha} \bar{A}_{\alpha \alpha}^{L} U_{L, i \alpha}^{\dagger}-H_{j i} U_{L, i \alpha} \bar{A}_{\alpha \alpha}^{L} U_{L, j \alpha}^{\dagger}\right)\right)- \\
& \left(\mathrm{i} f_{R}\left(H_{i j} U_{R, j \alpha} \bar{A}_{\alpha \alpha}^{R} U_{R, i \alpha}^{\dagger}-H_{j i} U_{R, i \alpha} \bar{A}_{\alpha \alpha}^{R} U_{R, j \alpha}^{\dagger}\right)\right) .
\end{aligned}
$$

\section{Transmission Plots \& Tables}

Here are plots of the transmission of the benzenes as calculated by GPAW along with plots and tables for the transmission of the macrocycles, i.e. fenestrane and annulenoannulene, used in the paper. It can be seen from the tables that GPAW predicts a higher degree of disagreement between the "sum rule" and the calculated transmissions for the spiders than that of gDFTB. Annulenoannulene based on the parallel configuration is the odd one out where GPAW predicts an almost perfect agreement. From the plots it can be seen that the resonances for the HOMOs and LUMOs all go to a maximum of one. Again here annulnoeannulene based on the parallel configuration sticks out, having a HOMO resonance of more than 1 . This means all of the molecules show the signs of coherence between the connections of the spider molecules, except annulenoannulene based on the parallel configuration that shows signs of incoherence.

Table 1: Transmission for meta fenestrane at the energy of the center of the HOMO-LUMO gap of the crossed connected molecule (at $0.425 \mathrm{eV}$ relative to the Fermi-energy for gDFTB and $0.545 \mathrm{eV}$ for GPAW.)

\begin{tabular}{|l|c|c|}
\hline \multirow{2}{*}{ Fenestrane } & \multicolumn{2}{|c|}{ Transmission } \\
\cline { 2 - 3 } & gDFTB $\left(10^{-6}\right)$ & GPAW $\left(10^{-4}\right)$ \\
\hline Direct & $0.0280(0 \%)$ & $0.2604(2 \%)$ \\
\hline Crossed & $8.1572(50 \%)$ & $7.1121(48 \%)$ \\
\hline Spider & $16.231(99 \%)$ & $6.0999(41 \%)$ \\
\hline 2 D + 2 C & $16.370(100 \%)$ & $14.745(100 \%)$ \\
\hline
\end{tabular}



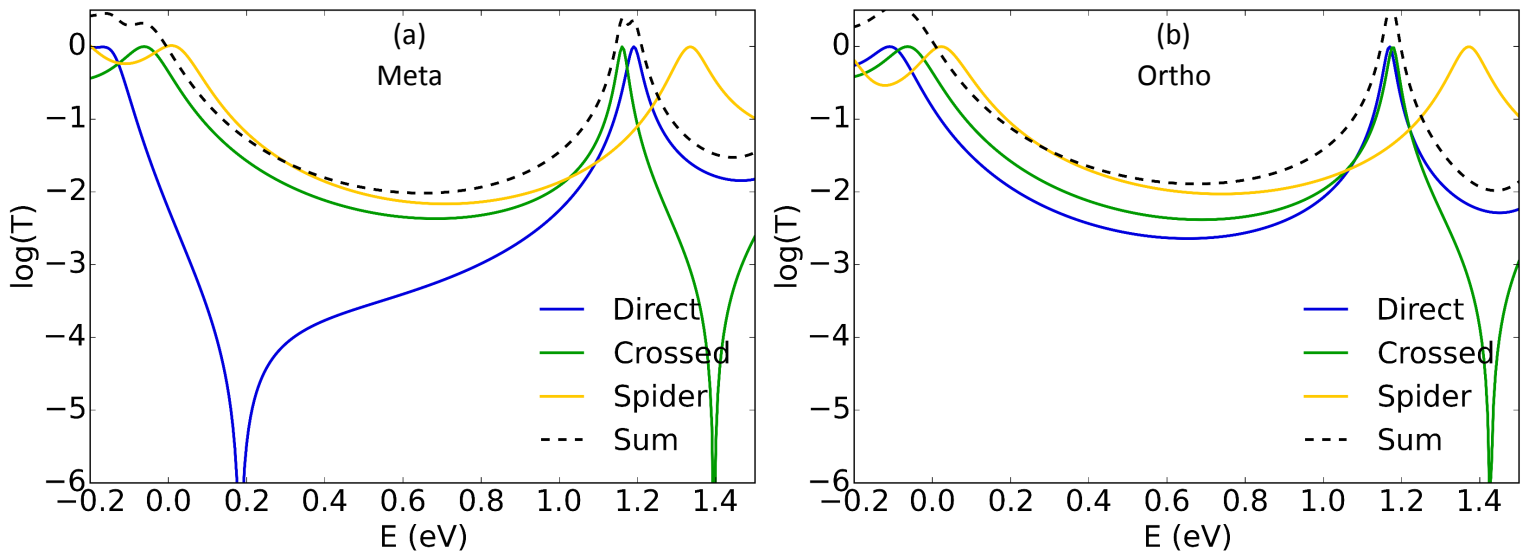

Figure S2: GPAW transmission of benzene based on the (a) meta-configuration and (b) ortho-configuration. The dotted line shows the transmission for the direct-connected molecule, the dashed it for the crossed-connected, and the solid for the spider-connected molecule.

Table 2: Transmission for ortho fenestrane at the energy of the center of the HOMO-LUMO gap of the crossed connected molecule (at $0.420 \mathrm{eV}$ relative to the Fermi-energy for gDFTB and $0.500 \mathrm{eV}$ for GPAW.)

\begin{tabular}{|l|c|c|}
\hline \multirow{2}{*}{ Fenestrane } & \multicolumn{2}{|c|}{ Transmission } \\
\cline { 2 - 3 } & gDFTB $\left(10^{-6}\right)$ & GPAW $\left(10^{-4}\right)$ \\
\hline Direct & $5.8338(22 \%)$ & $2.2216(8 \%)$ \\
\hline Crossed & $7.6221(28 \%)$ & $11.218(42 \%)$ \\
\hline Spider & $20.758(77 \%)$ & $7.3707(27 \%)$ \\
\hline $2 \mathrm{D}+2 \mathrm{C}$ & $26.912(100 \%)$ & $26.879(100 \%)$ \\
\hline
\end{tabular}

Table 3: Transmission for the parallel annulenoannulene at the energy of the center of the HOMO-LUMO gap of the crossed connected molecule (at $0.380 \mathrm{eV}$ relative to the Fermienergy for gDFTB and $0.315 \mathrm{eV}$ for GPAW.)

\begin{tabular}{|l|c|c|}
\hline \multirow{2}{*}{ Annulenoannulene } & \multicolumn{2}{|c|}{ Transmission } \\
\cline { 2 - 3 } & gDFTB $\left(10^{-5}\right)$ & GPAW $\left(10^{-3}\right)$ \\
\hline Direct & $1.5645(50 \%)$ & $3.2349(50 \%)$ \\
\hline Crossed & $0.0107(0 \%)$ & $0.0309(0 \%)$ \\
\hline Spider & $2.7261(87 \%)$ & $6.4462(99 \%)$ \\
\hline $2 \mathrm{D}+2 \mathrm{C}$ & $27.196(100 \%)$ & $3.1504(100 \%)$ \\
\hline
\end{tabular}



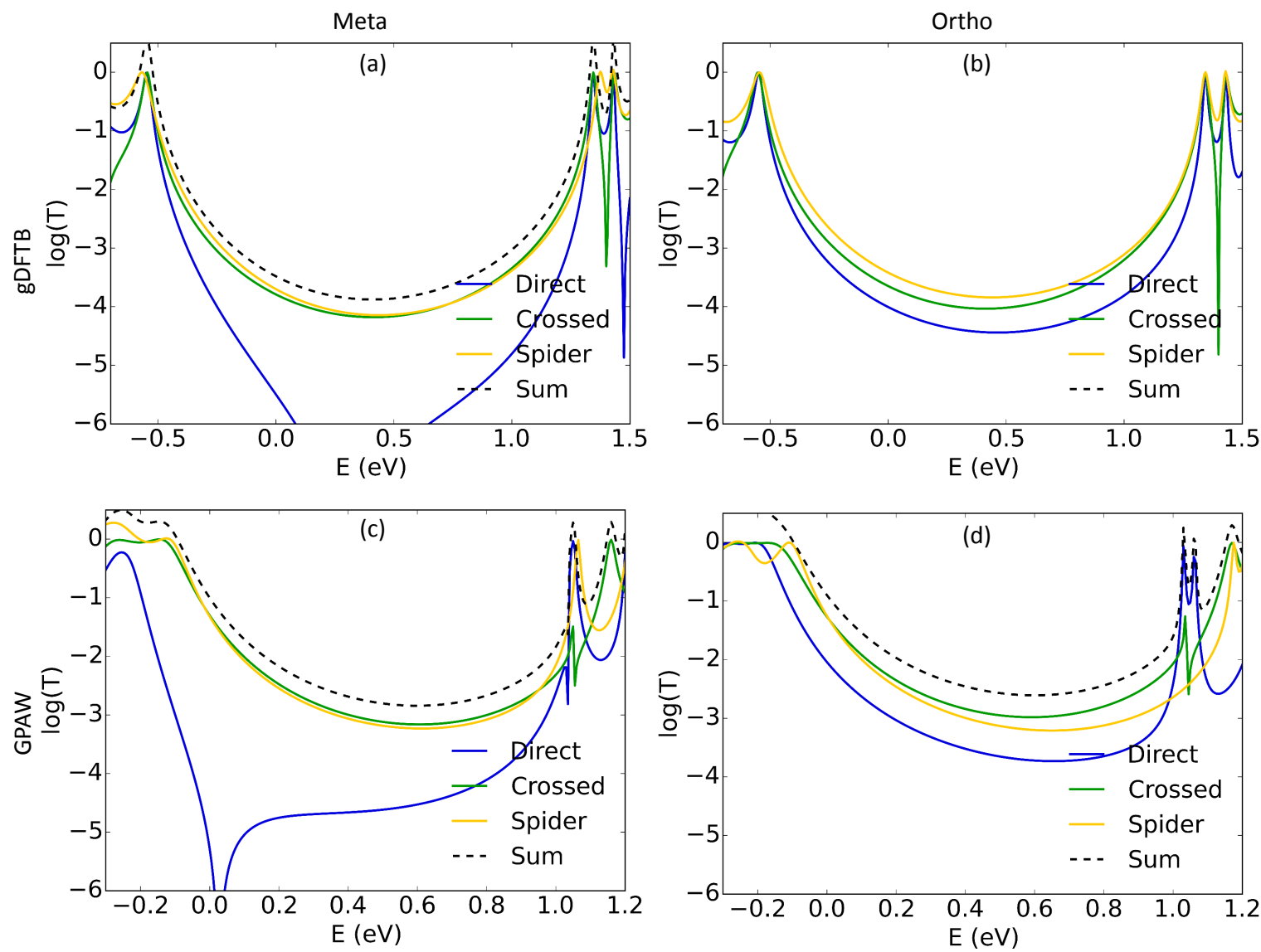

Figure S3: ( $\mathrm{a} \& \mathrm{~b})$ gDFTB and (c \& d) GPAW transmission of fenestrane based on the (a \& c) meta-configuration and (b \& e) ortho-configuration. The blue line shows the transmission for the direct-connected molecule, the green it for the crossed-connected, the yellow for the spider-connected molecule and the dashed black line is the transmission predicted by the sum rule.

Table 4: Transmission for the orthogonal annulenoannulene the energy of the center of the HOMO-LUMO gap of the crossed connected molecule (at $0.365 \mathrm{eV}$ relative to the Fermienergy for gDFTB and $0.315 \mathrm{eV}$ for GPAW.)

\begin{tabular}{|l|c|c|}
\hline \multirow{2}{*}{ Annulenoannulene } & \multicolumn{2}{|c|}{ Transmission } \\
\cline { 2 - 3 } & gDFTB $\left(10^{-8}\right)$ & GPAW $\left(10^{-5}\right)$ \\
\hline Direct & $5.9583(27 \%)$ & $5.3053(23 \%)$ \\
\hline Crossed & $4.9459(23 \%)$ & $6.2252(27 \%)$ \\
\hline Spider & $19.549(90 \%)$ & $6.2384(27 \%)$ \\
\hline 2 D $+2 \mathrm{C}$ & $21.808(100 \%)$ & $23.061(100 \%)$ \\
\hline
\end{tabular}



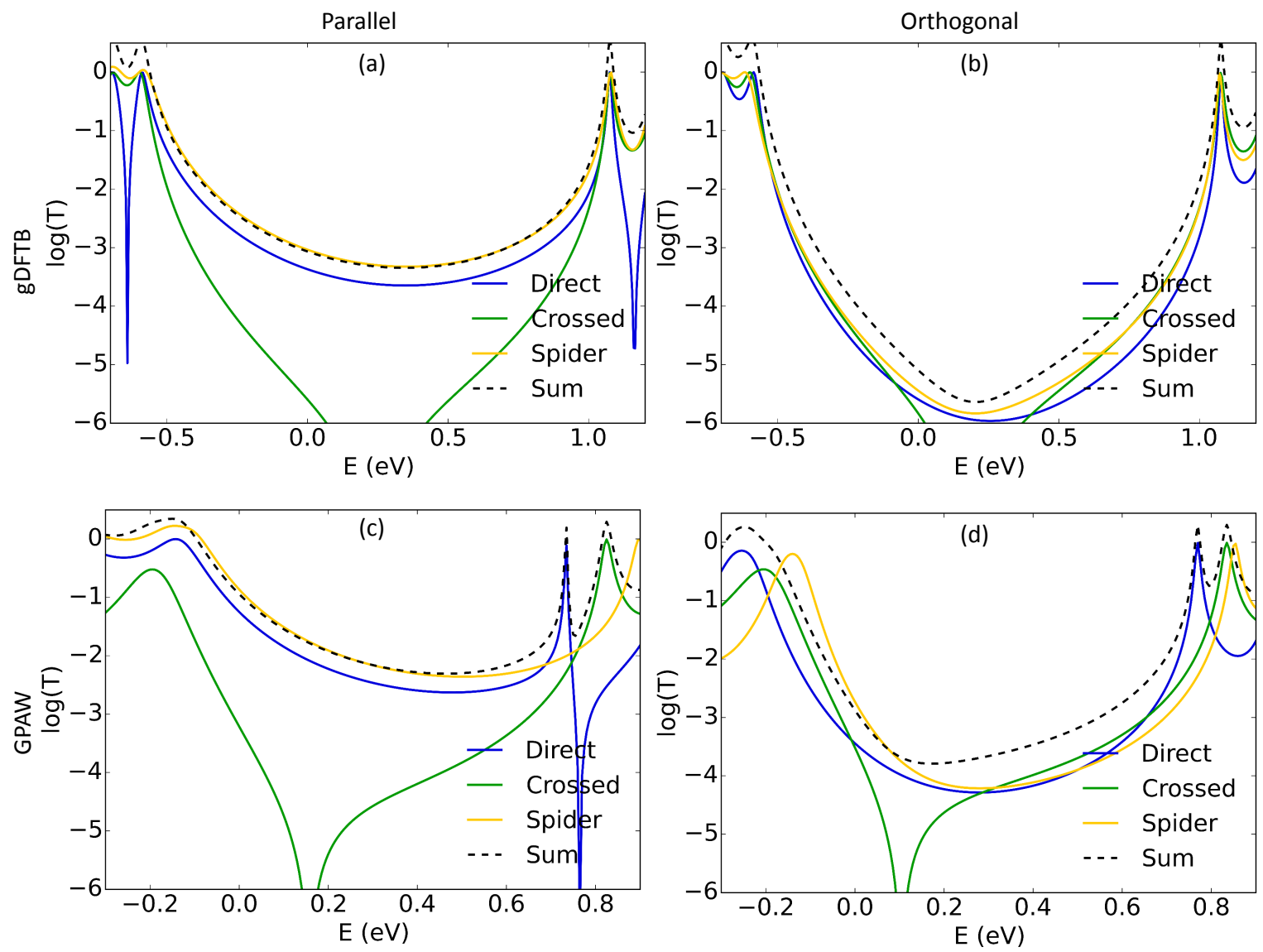

Figure S4: (a \& b) gDFTB and (c \& d) GPAW transmission of annulenoannulene based on the (a \& c) parallel-configuration and (b \& d) orthogonal-configuration. The blue line shows the transmission for the direct-connected molecule, the green it for the crossed-connected, the yellow for the spider-connected molecule and the dashed black line is the transmission predicted by the sum rule. 


\section{Local Currents}

Here are plots of the local transmissions at the Fermi energy of the macrocycles, i.e. fenestrane and annulenoannulene, used in the paper. Note that the arrows in the plots have been scaled so that the largest arrow in each plot is always of the same size, and thus the size of the transmission will have be gleaned from the relevant transmission plots in the previous section of the Supporting Information. Also note, that where the spider molecules had more than one dominant channel, the local transmission has been split into the contributions from each channel. In these cases this is always two, and occurs when can only exit from one of the connections on the opposite side of the molecule based on which connection from which it enters (in the sense that pathways to the other connection cannot occur without crossing a meta connection). The local transmission for the substituent channels may look like spatially

distinct pathways, as seen in Figures S6 and S7, but does not have to, as seen in Figure S8. 

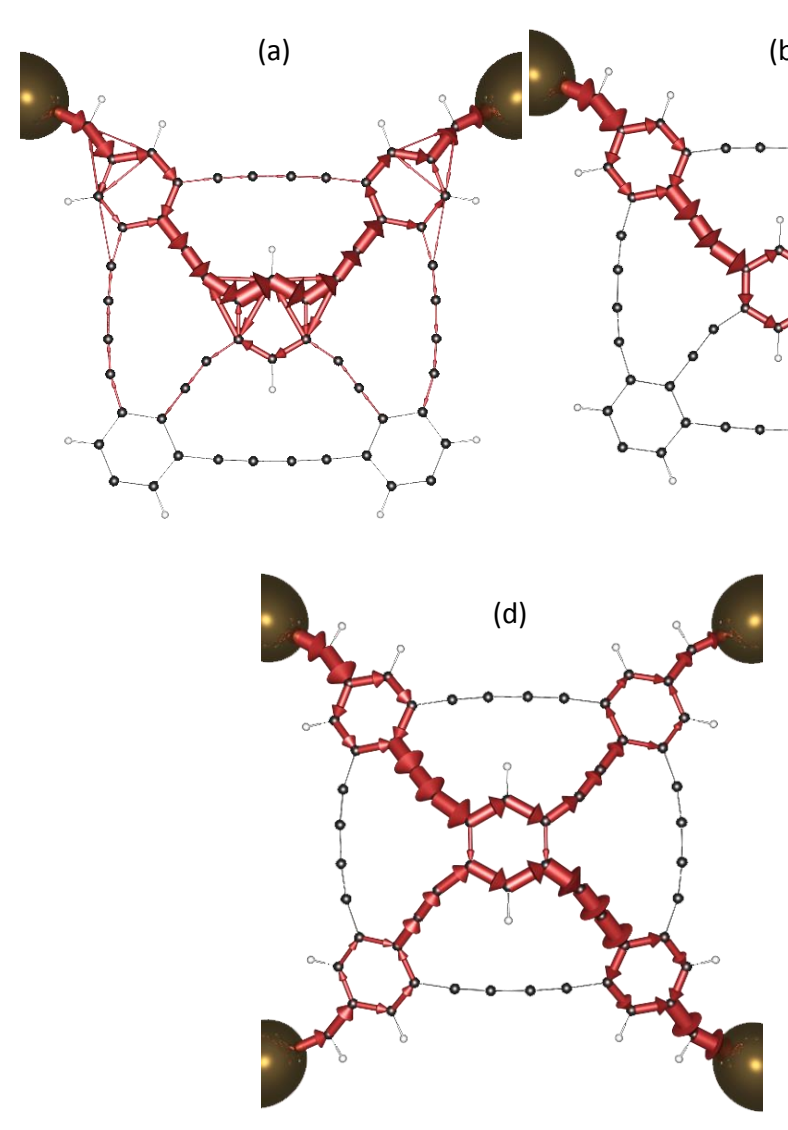

(b)
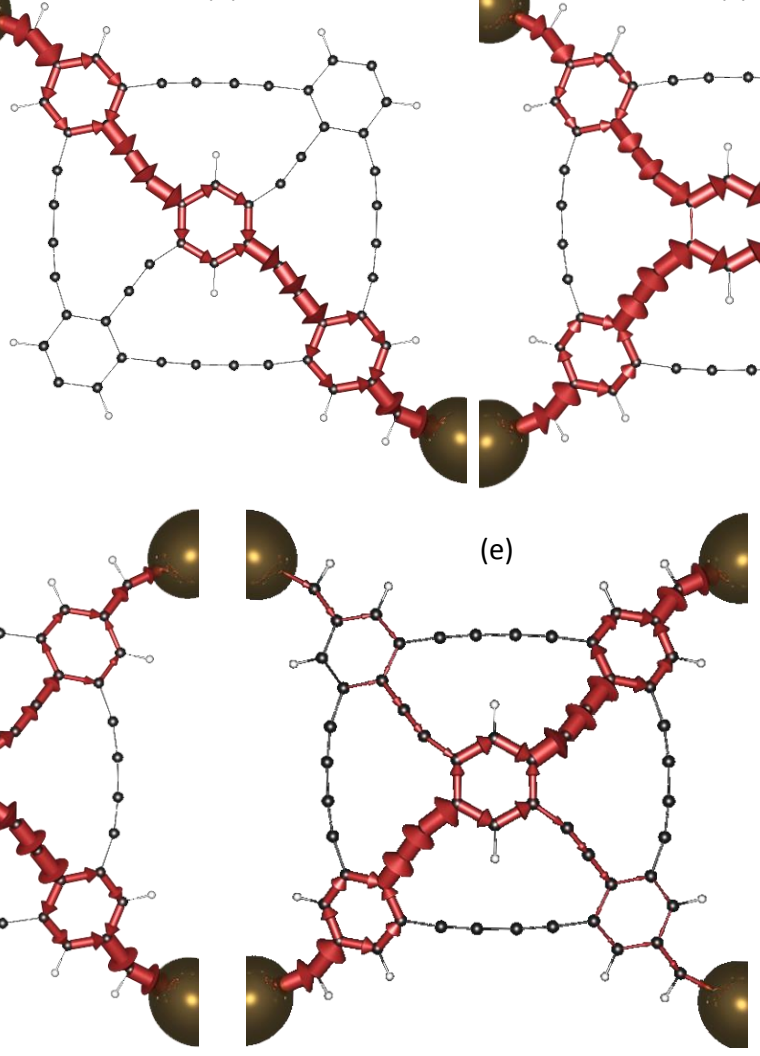

(c)

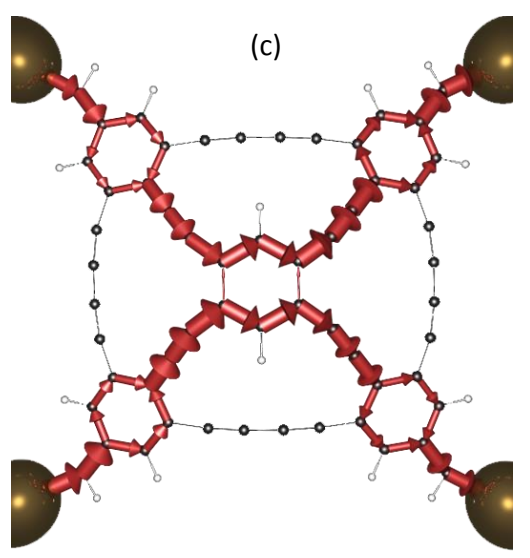

(e)

Figure S5: Local transmission elements of meta fenestrane plotted at the Fermi energy. (a) The direct connection, (b) the crossed connection. and (c) the spider connection. (d) and (e) shows the local transmission elements of the two dominant channels of the spider connection plotted at the Fermi energy. The binding linkers have been truncated and the non-binding linkers have been removed altogether for clarity.
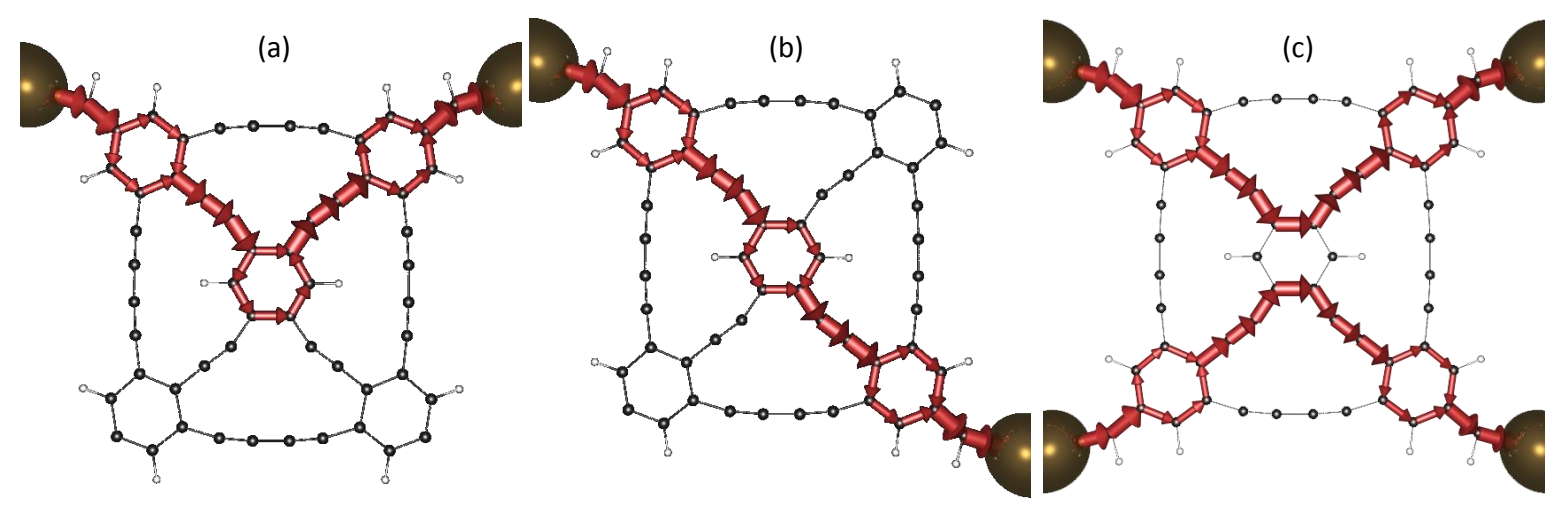

Figure S6: Local transmission elements of ortho fenestrane plotted at the Fermi energy. (a) The direct connection, (b) the crossed connection. and (c) the spider connection. This figure does not show the dominant channel of the fully connected molecule, since there is only one dominant channel. The binding linkers have been truncated and the non-binding linkers have been removed altogether for clarity. 

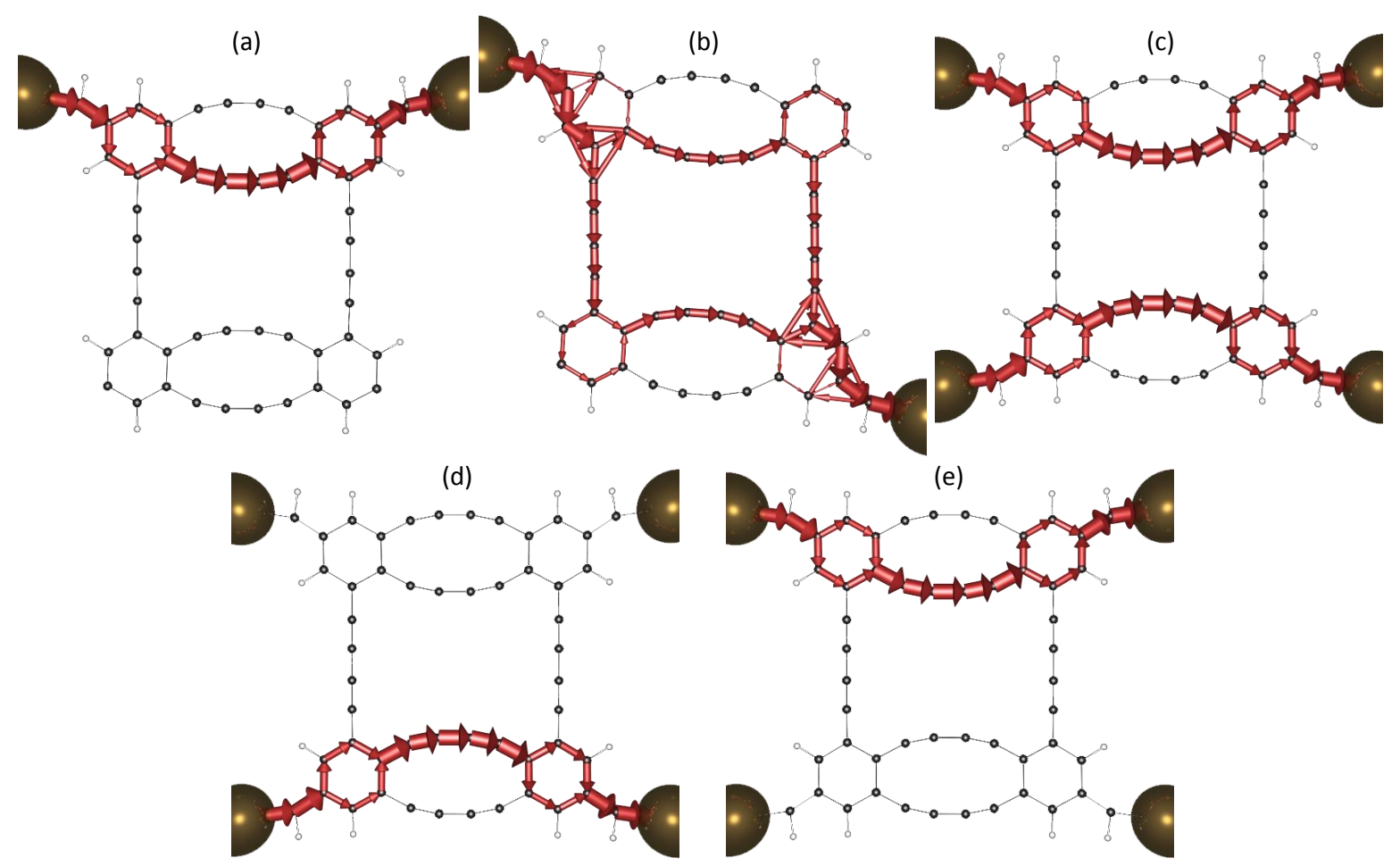

Figure S7: Local transmission elements of annulenoannulene in the parallel configuration plotted at the Fermi energy. (a) The direct connection, (b) the crossed connection. and (c) the spider connection. (d) and (e) shows the local transmission elements of the two dominant channels of the spider connection plotted at the Fermi energy. The binding linkers have been truncated and the non-binding linkers have been removed altogether for clarity. 

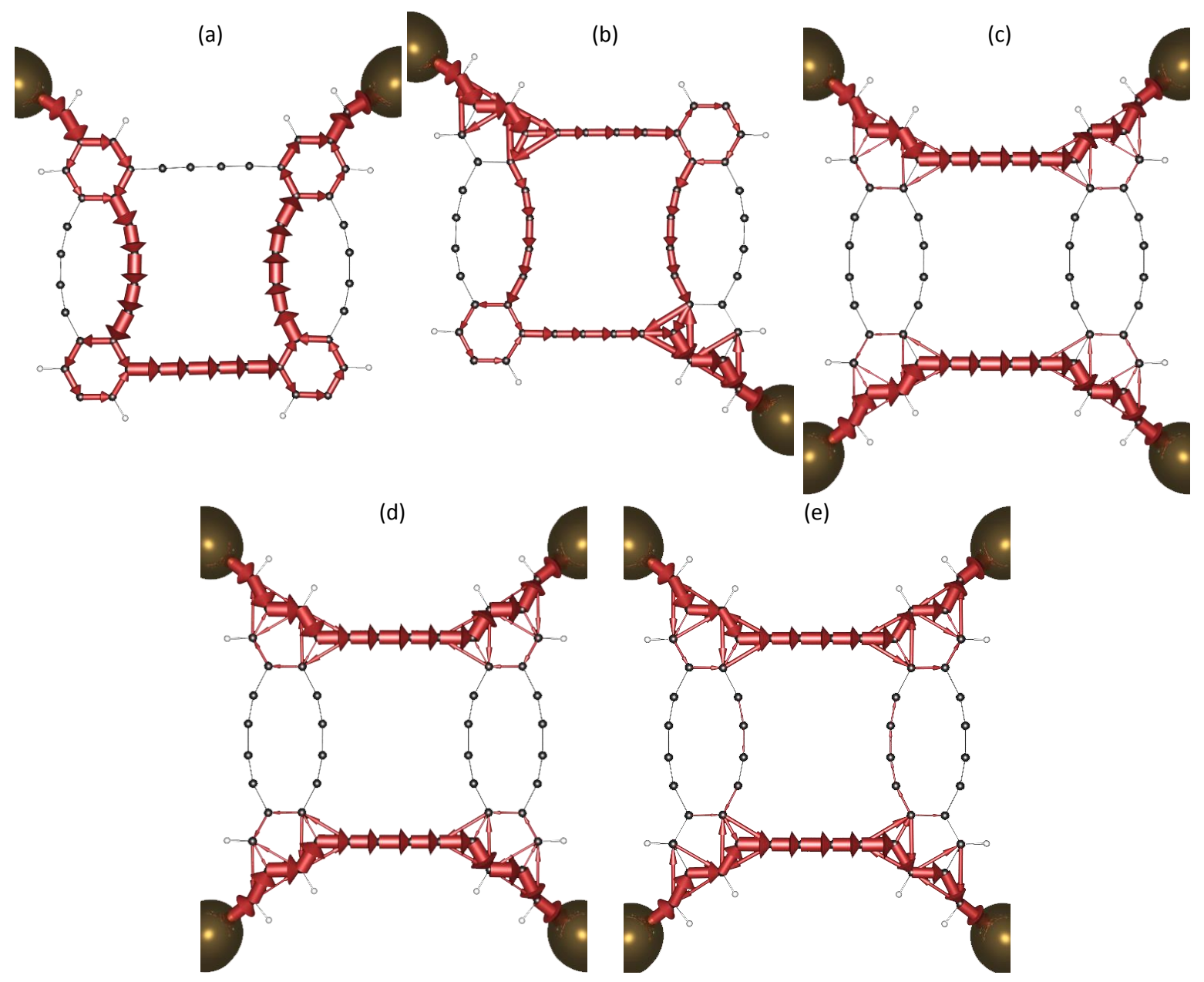

Figure S8: Local transmission elements of annulenoannulene in the orthogonal configuration plotted at the Fermi energy. (a) The direct connection, (b) the crossed connection. and (c) the spider connection. (d) and (e) shows the local transmission elements of the two dominant channels of the spider connection plotted at the Fermi energy. The binding linkers have been truncated and the non-binding linkers have been removed altogether for clarity. 


\section{References}

(1) Solomon, G. C.; Herrmann, C.; Hansen, T.; Mujica, V.; Ratner, M. A. Nat. Chem. 2010, 2, 223-228.

(2) Gagliardi, A.; Solomon, G. C.; Pecchia, A.; Frauenheim, T.; Di Carlo, A.; Hush, N. S.; Reimers, J. R. Phys. Rev. B 2007, 75, 174306. 